\title{
A nanophotonic InP/InGaAlAs optical amplifier integrated on a SOI waveguide circuit
}

\author{
M. Tassaert • S. Keyvaninia - D. Van \\ Thourhout • W.M.J. Green • Y. Vlasov • \\ G. Roelkens
}

Received: date / Accepted: date

\begin{abstract}
A small footprint integrated optical amplifier on Silicon-On-Insulator (SOI) is proposed in this article. By using a membrane active waveguide the optical confinement is maximized, leading to a compact device with high gain. Furthermore, the device is optically pumped through the silicon waveguide layer to optimally use the pump light. We show $8 \mathrm{~dB}$ gain in pulsed regime in a $100 \mu \mathrm{m}$ long device using a peak pump power of only $4.5 \mathrm{~mW}$. This is an important step towards a CMOS-compatible optical amplifier for intra-chip optical interconnects.
\end{abstract}

Keywords Silicon photonics - Optical amplifier · Heterogeneous integration · Optical interconnects

\section{Introduction}

The current tendency in high performance computing is directed towards parallelism on all levels. As the number of processor cores, executional units and transistors increases, the amount of data that needs to be sent across a chip increases as well. Electronics are reaching their data transfer limits however, so an alternative solution needs to be found. An integrated optical solution could provide for the necessary bandwidth for next-generation computing (Miller, 2000). However, to make an optical solution viable, its fabrication needs to be CMOS compatible to ensure cheap integration with the electronics circuits. The SiliconOn-Insulator (SOI) waveguide system would therefore be an excellent candidate. Furthermore, the high index contrast waveguides that can be achieved, show low loss and allow very tight bends, reducing the footprint of the photonic circuit (Bogaerts et al, 2008). However, to allow the realization of an integrated optical

M. Tassaert · S. Keyvaninia - D. Van Thourhout · G. Roelkens

Photonics Research Group - Ghent University/imec

Sint-Pietersnieuwstraat 41, 9000 Gent, Belgium

E-mail: martijn.tassaert@intec.ugent.be

W.M.J. Green · Y. Vlasov

IBM T.J. Watson Research Center, 1101 Kitchawan Road, Yorktown Heights, NY 10598, USA 
transmission system, also active components are required. Due to silicon's indirect band gap, creating these components remains a challenge on SOI. Our approach to address this problem, is by heterogeneously integrating an active III-V layer stack on top of the SOI waveguide circuit. This can be achieved by adhesive die-to-wafer bonding, using DVS-BCB as an intermediate adhesive between the SOI circuit and III-V layers (Roelkens et al, 2006). Several lasers and amplifiers have already been demonstrated using this method (Roelkens et al, 2010). However, in these devices the active III-V waveguide is at least $500 \mathrm{~nm}$ thick, to ensure efficient current injection in the device. This limits the maximal confinement of light one can achieve in the active layers, reducing the maximal gain and increasing the necessary device length, thereby increasing the power consumption. By reducing the thickness of the active III-V waveguide, one can leverage the high index contrast between the III-V and the surrounding air and DVS-BCB cladding to create III-V membrane waveguides with a much higher confinement in the active layers. In this article we propose a $100 \mathrm{~nm}$ thick membrane waveguide which is optically pumped through the underlying silicon waveguide layer.

\section{Device layout}

The device structure is schematically shown in figure 1(a). It consists of two silicon access waveguides which are coupled to a III-V membrane waveguide by two inverted taper couplers. These couplers are designed to support an adiabatic transition of the fundamental TE mode in the silicon waveguide to the fundamental TE mode in the III-V membrane waveguide. The couplers consist of a linearly tapered $220 \mathrm{~nm}$ thick silicon wire of $12 \mu \mathrm{m}$ long below the III-V waveguide, starting from a width of $700 \mathrm{~nm}$ and tapering down to a width of $100 \mathrm{~nm}$. The III-V waveguide is a strip waveguide with a width of $2 \mu \mathrm{m}$ and consists of four compressively strained $(0.85 \%)$ AlGaInAs quantum wells of $7 \mathrm{~nm}$ thick with five tensile strained AlGaInAs $(0.55 \%$, Q1.3) barriers of $10 \mathrm{~nm}$ sandwiched between two $10 \mathrm{~nm} \mathrm{InP}$ cladding layers. This quantum well stack has a bandgap wavelength of $1.6 \mu \mathrm{m}$. In figure 1(b), the mode profile in the membrane waveguide is shown for a wavelength of $1550 \mathrm{~nm}$, clearly displaying the high light intensity in the active layers. In this structure, a confinement of 0.22 can be achieved in the quantum wells. The device is connected to the outside world through two grating couplers, which have a coupling loss of $5 \mathrm{~dB}$ per coupler and a bandwidth of $70 \mathrm{~nm}$ (Taillaert et al, 2006).

\section{Fabrication}

Fabrication of the SOI waveguide circuit was done using $193 \mathrm{~nm}$ deep UV lithography and dry etching on a 8 inch SOI wafer in a CMOS pilot-line (Epixfab, 2004). After dicing of the wafer, the seperate dies are cleaned for 15 minutes in a Standard-Clean- 1 solution at $75 \mathrm{C}$. In the mean time, the III-V dies are prepared for bonding by removal of the InP and InGaAs sacrificial layers, using a $3 \mathrm{HCl}: \mathrm{H}_{2} \mathrm{O}$ solution and $1 \mathrm{H}_{2} \mathrm{SO}_{4}: 1 \mathrm{H}_{2} \mathrm{O}_{2}: 18 \mathrm{H}_{2} \mathrm{O}$ solution respectively. These layers are important to have a clean surface before bonding. After this, a diluted 


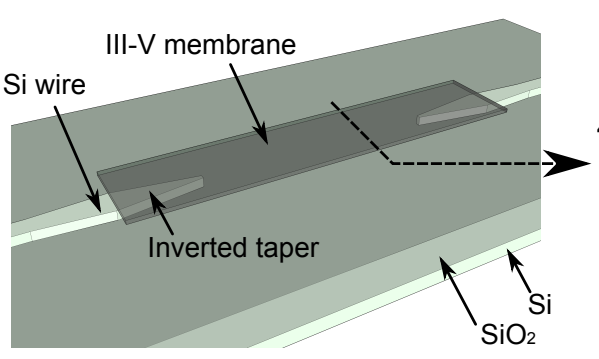

(a)

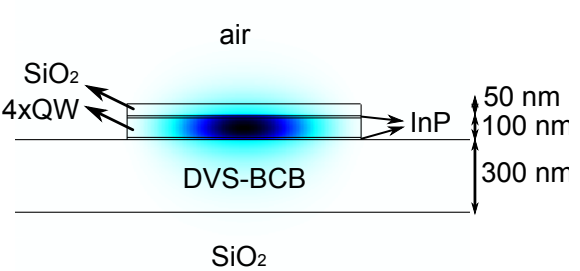

(b)

Fig. 1 (a) Schematic view of the device. It consists of two silicon access waveguides, a membrane III-V waveguide and two intermediate tapering sections. (b) The mode profile in the III-V membrane waveguide.

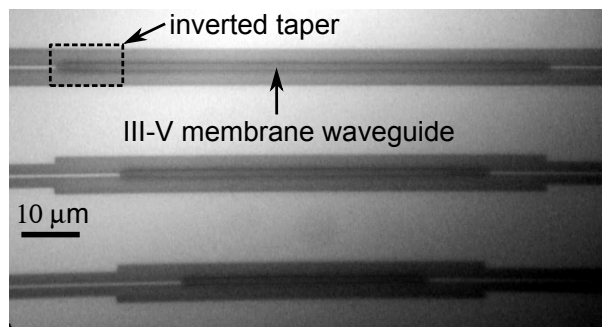

(a)

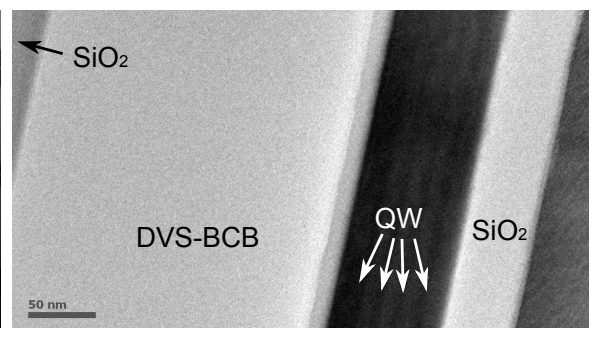

(b)

Fig. 2 (a) Optical microscopy image of the fabricated devices. (b) Transmission electron microscopy image of the III- $\mathrm{V}$ waveguide in the fabricated device.

DVS-BCB solution (1BCB:5Mes) is spincoated on the SOI die at $5000 \mathrm{rpm}$. After the evaporation of the mesitylene, the dies are brought into close contact and cured at $250 \mathrm{C}$ for one hour. Then, the InP substrate is removed using a combination of mechanical grinding and wet etching using a $3 \mathrm{HCl}: \mathrm{H}_{2} \mathrm{O}$ solution until the InGaAs etch stop layer is reached. After removal of the etch stop layer using a $1 \mathrm{H}_{2} \mathrm{SO}_{4}: 1 \mathrm{H}_{2} \mathrm{O}_{2}: 18 \mathrm{H}_{2} \mathrm{O}$ solution, a $50 \mathrm{~nm}$ thick $\mathrm{SiO}_{2}$ hard mask is deposited. This hard mask is patterned using contact lithography. Dry etching the hard mask and the III-V stack leaves the III-V membrane waveguides. Figure 2(a) shows a top down microscope image of the fabricated device. Figure 2(b) shows a transmission electron microscopy image of the quantum well layer stack bonded to the SOI waveguide circuit. From this figure, it can also be seen that the DVS-BCB bonding layer has a thickness of only $240 \mathrm{~nm}$ in the etched parts of the $220 \mathrm{~nm}$ thick silicon layer, which means that the bonding layer thickness is only $20 \mathrm{~nm}$ at this location.

\section{Characterization}

\subsection{Continuous Wave Experiment}

A continuous wave (CW) experiment was performed on these devices. A pump beam at a wavelength of $1545 \mathrm{~nm}$ was combined with a probe beam using a 99/1 combiner and sent through the device. By comparing the probe transmission 


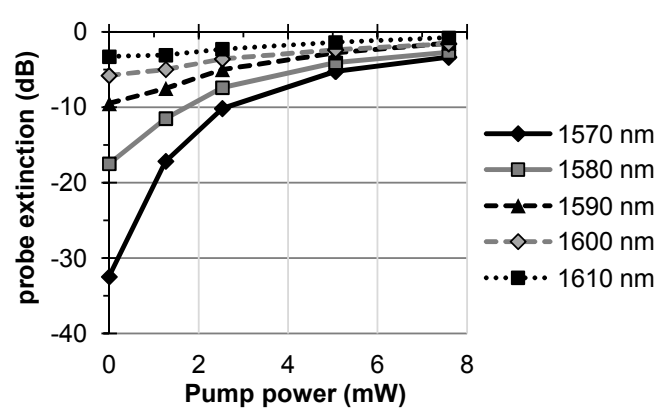

(a)

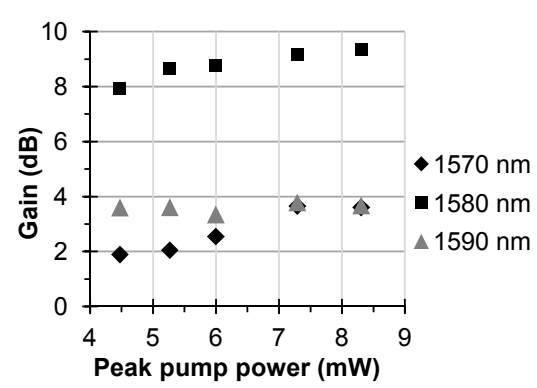

(b)

Fig. 3 (a) Measured CW gain in a $100 \mu \mathrm{m}$ long device. The pump power is the on-chip power, calculated by subtracting the $5 \mathrm{~dB}$ grating coupler loss from the measured pump power before the chip. (b) Net gain achieved a $100 \mu \mathrm{m}$ long device for the pulsed experiment.

through the device with the transmission through a reference silicon waveguide, the net gain in the III-V membrane waveguide can be extracted. In the experiment, both the pump power and the probe wavelength are varied. The results for a $100 \mu \mathrm{m}$ long device are shown in figure 3(a). When the pump power is increased, the absorption of the probe decreases as expected and eventually starts saturating. However, the point were we have net gain is not reached. This lack of net gain can be attributed to thermal effects, degrading the device performance. Both non-radiative recombination of the generated carriers (predominantly surface recombination) and free carrier absorption of the probe and pump beams are a cause for the heating of the device.

\subsection{Pulsed experiment}

To test our hypothesis that thermal effects cause the lack of gain, we have also performed a pulsed experiment. In this experiment, the CW pump laser is replaced by a directly modulated distributed feedback laser which also operates at $1545 \mathrm{~nm}$ and produces pump pulses of $0.3 \mu \mathrm{s}$ long with a period of $10 \mu \mathrm{s}$. This low-duty cycle pump signal avoids thermal effects. To boost the pump power, the pulses are send in a C-band erbium doped fiber amplifier (EDFA) and afterwards filtered by a band pass filter to avoid that the amplified stimulated emission (ASE) of the EDFA would pump the device. The pump is sent together with a CW probe beam in the device. Because of the pulsed character of the pump signal, the CW probe signal is modulated. After transmission through the device, the probe is amplified using a L-band EDFA and filtered in a band pass filter, which also removes the remaining pump. The resulting signal is then measured using an optical oscilloscope. The setup is shown in figure 4(a) and one of the measured probe traces is shown in figure 4(b). The achieved net gain is plotted in figure 3. From these results, it is clear that thermal effects must be the cause for the lack of gain in $\mathrm{CW}$ regime. A gain over $8 \mathrm{~dB}$ is achieved in pulsed regime, while in $\mathrm{CW}$ regime the device only barely reaches transparency for a comparable power. Furthermore, it seems that the gain is already saturated for the lowest used pump power at $1590 \mathrm{~nm}$, while it is still increasing a little for the shorter wavelengths. This increase is quite small 


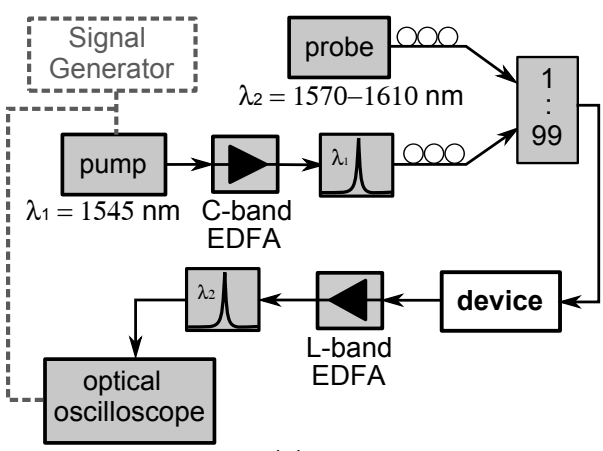

(a)

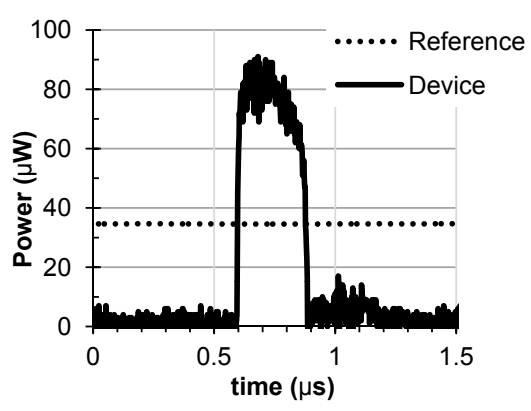

(b)

Fig. 4 (a) Measurement setup for the pulsed experiment. (b) Trace of the resulting pulse profile measured by the optical oscilloscope. This measurement is for a $1570 \mathrm{~nm}$ probe beam and a peak pump power of $7.29 \mathrm{~mW}$. The dashed line shows the transmitted power through the reference waveguide.

however, because of absorption saturation of the pump beam. To increase the gain even more, one should move to a shorter pump wavelength, but in the current setup the limited bandwidth of the grating couplers limits the separation between pump and probe wavelength.

\subsection{Thermal analysis}

To achieve CW gain, the thermal performance of the device needs to be improved. To evaluate the current design, the thermal resistance of the device was simulated using COMSOL (figure 5(a)). It seems that the device has a thermal resistance of $9 \mathrm{~K} / \mathrm{mW}$. This high value can be attributed to both the low thermal conductivity of the $240 \mathrm{~nm}$ thick DVS-BCB bonding layer $(0.3 \mathrm{~W} /(\mathrm{m} \cdot \mathrm{K}))$ and the $2 \mu \mathrm{m}$ thick buried oxide layer $(1.27 \mathrm{~W} /(\mathrm{m} \cdot \mathrm{K}))$ which forms the cladding for the silicon waveguides (Van Campenhout, 2007). Furthermore, the heat is dissipated in a small volume, which has a small contact area with the SOI die. As we cannot change the bonding material and buried oxide thickness easily, we can only improve the

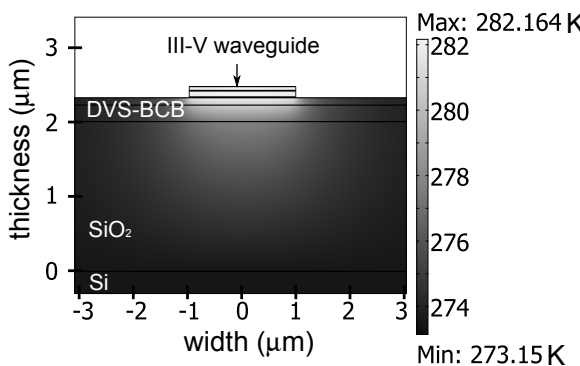

(a)

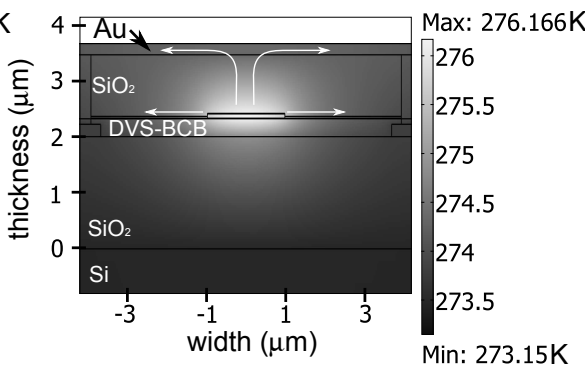

(b)

Fig. 5 (a) Thermal simulation in COMSOL of the fabricated device for a heat dissipation of $1 \mathrm{~mW}$. (b) Thermal simulation in COMSOL of an improved design with shallow etch and heat spreader for a heat dissipation of $1 \mathrm{~mW}$. 
thermal behaviour by increasing this contact area. There are two strategies to do this: first of all, the III-V membrane waveguide could be shallow etched instead of completely etched. This allows for a heat flow towards the shallow etched parts of the III-V waveguide. This shallow etch could at the same time also decrease the scattering loss in the waveguide and the amount of non-radiative recombination due to a reduction of surface recombination. A second strategy is the use of a thick $\mathrm{SiO}_{2}$ overcladding with a gold layer on top, which acts as a heat spreader. The simulation shows that the combination of these strategies reduces the thermal resistance of the structure by a factor of three to $3 \mathrm{~K} / \mathrm{mW}$ (see figure $5(\mathrm{~b})$ ).

\subsection{Conclusion}

In summary, we have proposed to use thin III-V membranes to create an efficient integrated optical amplifier on SOI. The first fabricated devices show no CW gain however, which seems to be caused by thermal effects. The measured net gain of $8 \mathrm{~dB}$ in pulsed mode at a peak pump power of only $4.5 \mathrm{~mW}$ shows the potential of this device. To address the thermal quenching of the gain, we have proposed to use a shallow etched waveguide instead and to add a heat spreader on top. In future work, we will implement these solutions and use horizontal couplers instead of grating couplers to be able to pump the devices at shorter wavelengths as well.

Acknowledgements Martijn Tassaert acknowledges the Bijzonder Onderzoeksfonds (BOF) for a doctoral grant.

\section{References}

Bogaerts W, Liu L, Selvaraja S, Brouckaert J, Taillaert D, Vermeulen D, Roelkens G, Van Thourhout D, Baets R (2008) Silicon nanophotonic waveguides and their applications. In: Asia-Pacific Optical Communications (APOC), China, pp 71,341O-71,341O-13

Epixfab (2004) www.epixfab.eu

Miller D (2000) Optical interconnects to silicon. IEEE Journal on Selected Topics in Quantum Electronics 6:1312-1317

Roelkens G, Brouckaert J, Van Thourhout D, Baets R, Notzel R, Smit M (2006) Adhesive bonding of inp/ingaasp dies to processed silicon-on-insulator wafers using dvs-bis-benzocyclobutene. Journal of Electrochemical Society 153(12):G1015-G1019

Roelkens G, Liu L, Liang D, Jones R, Fang A, Koch B, J B (2010) Iii-v/silicon photonics for on-chip and inter-chip optical interconnects. Laser \& Photonics reviews

Taillaert D, Van Laere F, Ayre M, Bogaerts W, Van Thourhout D, Bienstman P, Baets R (2006) Grating couplers for coupling between optical fibers and nanophotonic waveguides. Japanese Journal of Applied Physics Part 1-Regular Papers Brief Communications 45:60716077

Van Campenhout J (2007) Thin-film microlasers for the integration of electronic and photonic integrated circuits. PhD thesis 